\title{
Clostridium difficile infection secondary to ileostomy closure
}

\author{
Elie Chouillard, Marc-Anthony Chouillard, Nader El Kary, Belinda De Simone, Andrew A. Gumbs \\ Département de Chirurgie Digestive, Centre Hospitalier Intercommunal, de POISSY/SAINT-GERMAIN-EN-LAYE, Poissy \\ 78300, France.
}

Correspondence to: Prof. Elie Chouillard, Département de Chirurgie Digestive, Centre Hospitalier Intercommunal, de POISSY/ SAINT-GERMAIN-EN-LAYE, 10 rue du Champ Gaillard, Poissy 78300, France. E-mail: chouillard@yahoo.com

How to cite this article: Chouillard E, Chouillard MA, Kary NE, De Simone B, Gumbs AA. Clostridium difficile infection secondary to ileostomy closure. Mini-invasive Surg 2021;5:9. http://dx.doi.org/10.20517/2574-1225.2020.108

Received: 24 Nov 2020 First Decision: 21 Dec 2020 Revised: 23 Dec 2020 Accepted: 12 Jan 2021 Published: 9 Mar 2021

Academic Editors: Giulio Belli, Biondi Alberto Copy Editor: Yue-Yue Zhang Production Editor: Jing Yu

\begin{abstract}
Protective ileostomy may be a risk factor for the development of Clostridium difficile (CD) infection (CDI). In the postoperative period signs of CDI may be particularly difficult to differentiate from intra-abdominal sepsis. Presented here are 2 cases that developed CDI after ileostomy reversal. Two patients who underwent low anterior resections after neoadjuvant chemoradiation with protective ileostomy developed fever, leukocytosis and elevated serum C-reactive protein (CRP) levels. The first patient also had negative CD stool toxins and his signs were so severe that he underwent a negative diagnostic laparoscopy and re-creation of ileostomy. The second patient who presented in a similar fashion was more fortunate in that her CD stool toxin was positive and she was treated successfully with oral vancomycin. CDI after ileostomy reversal after low anterior resection can be difficult to diagnose. In the first patient, the situation was so misleading that diagnostic laparoscopy was required. Outcome was eventually favorable in both cases. CDI must be high on the list of differential diagnoses for febrile patients with a leukocytosis and elevated CRP level even in the setting of negative CD stool toxins. Prophylactic intravenous metronidazole and/or vancomycin enemas should be considered prior to colorectal surgery when a protective ileostomy is likely.
\end{abstract}

Keywords: Clostridium difficile, stoma, cancer, rectal, laparoscopy, surgery, pseudomembranous colitis 


\section{INTRODUCTION}

Proximal fecal diversion through a loop ileostomy is commonly used to protect colorectal anastomosis. Patients undergoing total mesorectal excision (TME) for rectal cancer are at higher risk of developing an anastomotic leak ${ }^{[1]}$. Diverting stomas were found to decrease both the clinical anastomotic leak rate and the risk of re-operation in patients undergoing low anterior resection or $\mathrm{TME}^{[2]}$. The temporary stoma is usually closed 8 to 12 weeks after surgery, or even earlier ${ }^{[3]}$ when there are no clinical or radiological signs of leak. Clostridium difficile infection (CDI) is a major cause of hospital-acquired infection that continues to increase in incidence and severity among hospitalized patients ${ }^{[4,5]}$. Symptoms range from mild diarrhea to fulminant colitis causing severe sepsis, toxic megacolon, and even death. The major risk factors for acquiring CDI are previous antibiotic exposure, severe underlying disease, older age, and immune suppression $^{[4,5]}$. In this paper, we report on an unusual presentation of CDI in 2 patients who had an elective reversal of ileostomy after TME for rectal cancer. The initial presentation of CDI mimicked more common causes of postoperative intra-abdominal sepsis.

\section{CASE REPORT}

\section{Case \# 1}

A 47-year-old woman, with an unremarkable past medical history was diagnosed with low rectal adenocarcinoma. She underwent trans-anal $\mathrm{TME}^{[6]}$ with diverting loop ileostomy 10 weeks after the completion of a neoadjuvant treatments, including 45-Gr external beam radiotherapy and oral 5-fluorouracil. Her postoperative course was uneventful. Final pathology diagnosed a ${ }_{\mathrm{p}} \mathrm{T}{ }_{\mathrm{p}} \mathrm{No}$ adenocarcinoma with an Ro resection. Fourteen days after resection, the patient underwent ileostomy closure after digital exam, endoscopy, and a computerized tomography (CT) scan showed no evidence for an anastomotic leak. She received one dose of intravenous antibiotics (cefuroxime $1 \mathrm{~g} \mathrm{IV}$ ) at the induction of anesthesia. The immediate postoperative outcome was uneventful.

However, 3 weeks postoperatively, the patient started having lower abdominal pain and severe diarrhea with over 10 bowel movements per day. Physical examination revealed a fever at $38.5^{\circ} \mathrm{C}$, a heart rate of $92 \mathrm{BPM}$, and blood pressure at 110/70 $\mathrm{mmHg}$. Abdominal examination was within normal limits and did not reveal any signs of superficial surgical site infections. Gynecological evaluation was negative for sepsis. Serum blood tests revealed a leukocytosis with white blood count of $13,500 / \mathrm{mm}^{3}$ and an elevated C-reactive protein (CRP) at $132 \mathrm{mg} / \mathrm{L}$. Stool testing for Clostridium difficile toxin was negative. As symptoms worsened with persistent fever, a pelvic magnetic resonance imaging (MRI) scan was performed and revealed evidence suggestive for a leak of the colo-anal anastomosis [Figure 1].

A diagnostic laparoscopy was performed. More than 2 liters of clear liquid was found in the peritoneal cavity that ultimately tested negative for bacteria, fungus, creatinine, bilirubin, or amylase. There were no signs of intestinal perforation or ureteral injury; however, the colon was hypervascularized, thickened, and dilated. A loop ileostomy was again performed.

Postoperatively, stool cultures became positive for Clostridium difficile (CD). Intravenous metronidazole was administered for $48 \mathrm{~h}$ then orally for 10 more days. Clinical improvement occurred rapidly.

\section{Case \# 2}

A 64-year-old man, with a past medical history of hypertension, type 2 diabetes mellitus, and coronary artery disease, was diagnosed with low rectal adenocarcinoma. He underwent trans-anal TME with diverting loop ileostomy, 11 weeks after neoadjuvant treatment, including 45-Gr external beam radiotherapy and oral 5-fluorouracil. His initial postoperative course was uneventful. Pathology report showed a ${ }_{\mathrm{p}} \mathrm{T} 3_{\mathrm{p}} \mathrm{No}$ adenocarcinoma with an Ro resection. Eight weeks after surgery, the patient had 

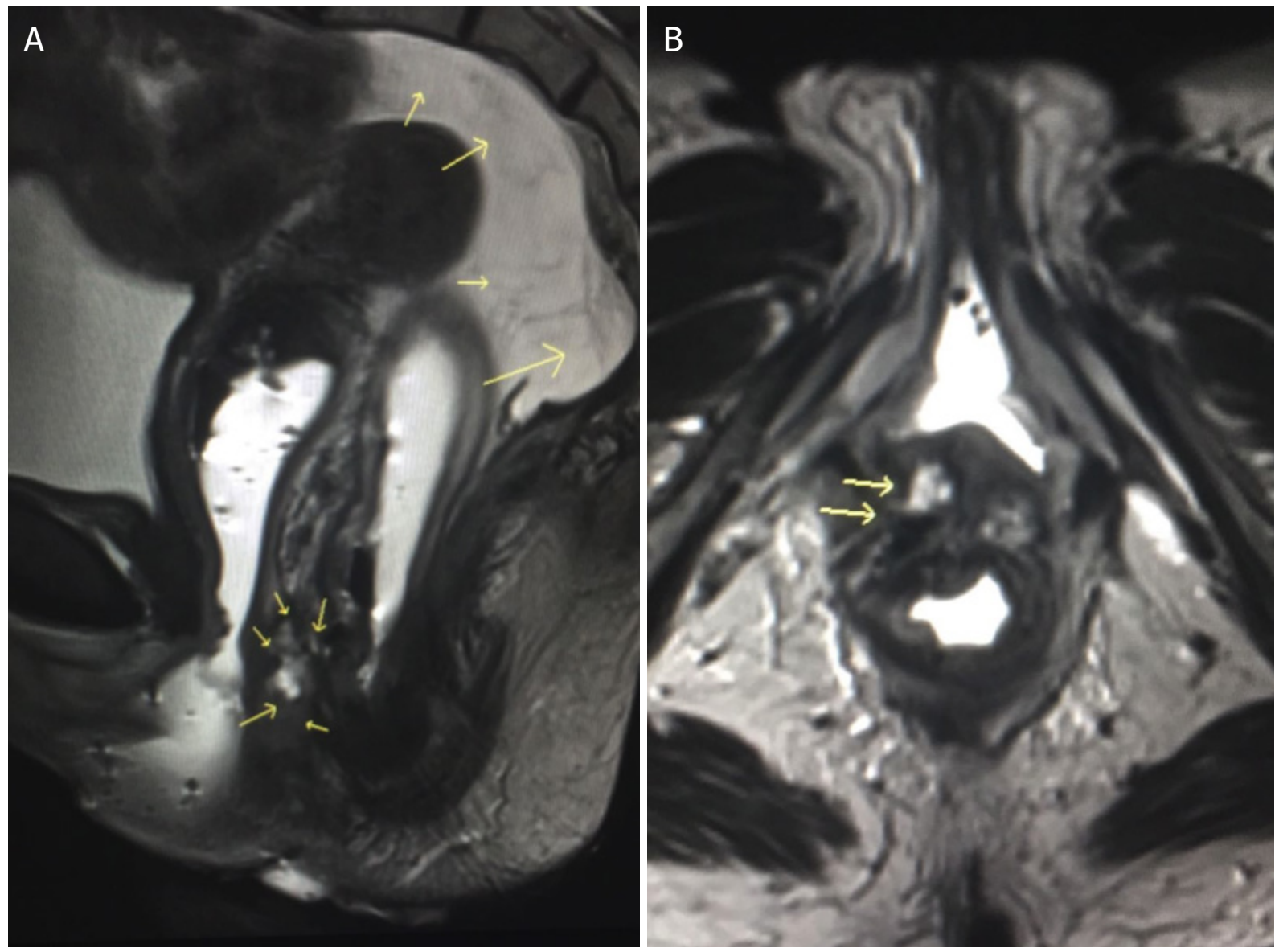

Figure 1. Pelvic magnetic resonance imaging (MRI) scan in a 47-year old woman with pain and febrile diarrhea 2 weeks after total mesorectal excision (TME) with colo-anal anastomosis: (A) small arrows in a circle at the bottom of the image show fluid next to the colo-anal anastomosis evoking leak. Large arrow on the right of the image shows massive intra-peritoneal fluid. Note the concern for colonic wall thickening (horizontal arrow); (B) small arrows show what was considered as extravasation of contrast in the vicinity of the colo-anal anastomosis.

ileostomy closure after a normal pelvic CT scan with contrast. He received one dose of prophylactic antibiotics (cefuroxime $1 \mathrm{~g} \mathrm{IV}$ ) at the induction of anesthesia. The immediate postoperative outcome was uneventful. Ten days postoperatively, the patient started having diffuse abdominal pain and watery diarrhea with 6 to 7 bowel movements per day. Physical examination revealed fever at $39{ }^{\circ} \mathrm{C}$, tachycardia at 112 $\mathrm{BPM}$, and hypertension at $170 / 100 \mathrm{mmHg}$. Abdominal examination, including digital rectal examination, was normal. Blood chemistries were consistent with acute renal failure (blood urea nitrogen at $61 \mathrm{mg} / \mathrm{dL}$; creatinine at $2.9 \mathrm{mg} / \mathrm{dL}$ ), leukocytosis with white blood count at $23,000 / \mathrm{mm}^{3}$, and increased CRP at $252 \mathrm{mg} / \mathrm{L}$. Stool testing for toxin-producing CD was positive. Treatment was with $2 \mathrm{~g}$ of oral vancomycin for 10 days. The patient's renal function fully recovered without the need for dialysis. Neither patient received adjuvant chemotherapy.

\section{DISCUSSION}

Symptoms of CD colitis such as pain, diarrhea, and increased CRP may be indistinguishable from other causes of intra-abdominal sepsis (i.e., anastomotic leak, pelvic abscess, or iatrogenic bowel injury). This could cause delay in the diagnosis of CDI, which could be fatal ${ }^{[7]}$. In the literature, we could only find a few papers that reported CDI after closure of ileostomy ${ }^{[7-11]}$. In some reported cases, as in our first case, the presentation was confusing, and the diagnosis was delayed. In another case, the disease was even much more severe (fulminant colitis), and the patient deteriorated quickly and died following an emergency total colectomy ${ }^{[7]}$. 
Three studies looked more closely into the incidence of CD after ileostomy closure ${ }^{[12-14]}$. Hussain et al. ${ }^{[12]}$ prospectively evaluated 20 patients undergoing ileostomy reversal. Two stool samples were collected before and after the procedure and tested for $\mathrm{CD}$ and toxins $\mathrm{A}$ and $\mathrm{B}$. None of the patients had positive tests preoperatively. Two of the 20 patients had asymptomatic postoperative CD colonization (10\%), while one patient developed clinical CDI with positive toxins (5\%). Randall et al ${ }^{[13]}$ retrospectively analyzed patients who had ileostomy closure and subsequent CDI. Six (4.2\%) of the 143 patients who had ileostomy reversal developed CDI. In a retrospective large population-based analysis (2004-2008) in the US, Wilson et al. ${ }^{[14]}$ found the incidence of CDI after ileostomy closure to be $1.6 \%$.

There is no clear explanation yet for the high rate of CDI after ileostomy closure. Theoretically, CD could colonize the small bowel, with many studies reporting symptomatic enteritis. Animal studies have shown that excluded colons undergo mucosal and muscular atrophy with derangement in the intestinal immune system. The exclusion of the colon could change the unique microbial ecosystem in the large bowel and favor the growth of CD. When the stoma is closed, the spores could get reactivated and enter a growth phase leading to clinical infection.

We suppose that the prophylactic antibiotics administered at the induction of anesthesia at the index operation may have triggered the CDI in our 2 cases. Previous studies have reported that the risk of subsequent CDI was 5.9-fold higher among patients colonized with toxigenic CD upon hospital admission as compared to non-colonized patients ${ }^{[15]}$. In our protocol, patients are tested for CD colonization before all colorectal resections. Both patients in our study were negative preoperatively. Besides antibiotics as well-known risk factors for $\mathrm{CDI}^{[16]}$, other incriminating factors include previous hospitalization within 3 months ${ }^{[15]}$, chemotherapy within the previous 8 weeks ${ }^{[17]}$, or even gastric acid suppression with proton pump inhibitors (PPIs) ${ }^{[18]}$.

Rubio-Perez et al. ${ }^{[19]}$ reported a significant association between CDI and delayed ileostomy reversal (of greater than 6 months), with the reported dysfunctional time ranging from 9 to 15 months. Our two patients underwent ileostomy closure less than 2 months after the first surgery. Neither patient received PPIs, and both had stopped their oral chemotherapy more than 3 months earlier. A meta-analysis published in 2017 found that the incidence of CDI after ileostomy reversal was 1.8\%. It also suggested that probiotics should be considered, PPIs avoided, and rectal swabs considered in high-risk patients, and that when possible ileostomy closure should be scheduled within 6 months ${ }^{[20]}$.

Despite its low incidence, the clinical presentation of CDI may be indistinguishable from the usual postoperative state. Therefore, diagnosis could be challenging. Since fulminant cases are known to occur, clinicians must consider this condition in the differential diagnosis. Prompt evaluation is warranted in patients undergoing ileostomy reversal who present with severe diarrhea and abdominal pain. Clinicians should be aware of the risk factors for CDI. Systematic preoperative testing of colonization with CD should be encouraged. We also recommend reducing the use of unwarranted antibiotics and PPIs.

Although neither of these patients required adjuvant chemotherapy, and the best timing of ileostomy closure during or after adjuvant treatments has not been well established, one should consider early ileostomy reversal where appropriate, even if it does not seem to completely prevent CDI. Considering the nature of the topic and question, the highest level evidence that can potentially be achieved in this context is from case-control studies (level 3) and meta-analysis of observational studies (level 2-3). Notably, a meta-analysis did not see any difference in outcomes whether ileostomies were reversed during or after adjuvant treatments ${ }^{[21]}$. Prophylactic use of vancomycin enemas in the excluded colons prior to ileostomy closure is an option to be further evaluated ${ }^{[22]}$. Additionally, metronidazole should potentially be added to the preoperative regimen when a protective ileostomy is envisioned. 


\section{DECLARATIONS}

\section{Authors' contributions}

Data analysis and interpretation, drafting of manuscript, and editing: Chouillard E, Gumbs AA

Performed data acquisition and data analysis: Chouillard MA, Kary NE

Provided administrative support and editing: De Simone B

\section{Availability of data and materials}

Not applicable.

\section{Financial support and sponsorship}

None.

\section{Conflicts of interest}

All authors declared that there are no conflicts of interest.

\section{Ethical approval and consent to participate}

Not applicable.

\section{Consent for publication}

Not applicable.

\section{Copyright}

(C) The Author(s) 2021.

\section{REFERENCES}

1. Blumetti J, Abcarian H. Management of low colorectal anastomotic leak: Preserving the anastomosis. World J Gastrointest Surg 2015;7:378-83.

2. Weston-Petrides GK, Lovegrove RE, Tilney HS, et al. Comparison of outcomes after restorative proctocolectomy with or without defunctioning ileostomy. Arch Surg 2008;143:406-12.

3. Danielsen AK, Park J, Jansen JE, et al. Early Closure of a Temporary Ileostomy in Patients With Rectal Cancer: A Multicenter Randomized Controlled Trial. Ann Surg 2017;265:284-90.

4. Czepiel J, Dróżdż M, Pituch H, et al. Clostridium difficile infection: review. Eur J Clin Microbiol Infect Dis 2019;38:1211-21.

5. Burke KE, Lamont JT. Clostridium difficile infection: a worldwide disease. Gut Liver 2014;8:1-6.

6. Chouillard E, Chahine E, Khoury G, et al. NOTES total mesorectal excision (TME) for patients with rectal neoplasia: a preliminary experience. Surg Endosc 2014;28:3150-7.

7. Abe I, Kawamura YJ, Sasaki J, Konishi F. Acute fulminant pseudomembranous colitis which developed after ileostomy closure and required emergent total colectomy: a case report. J Med Case Rep 2012;6:130.

8. Almerie MQ, Culverwell A, Mahon C. Clostridium difficile infection after ileostomy closure mimicking anastomotic leak. BMJ Case Rep 2015;2015:bcr2015210112.

9. Shen B, Remzi FH, Fazio VW. Fulminant Clostridium difficile-associated pouchitis with a fatal outcome. Nat Rev Gastroenterol Hepatol 2009;6:492-5.

10. Nair MS, Uzzaman MM, Chung J, Navaratnam R. Chylous ascites secondary to pseudomembranous colitis following ileostomy reversal fashioned for low anterior resection. Int J Colorectal Dis 2010;25:791-2.

11. Fashandi AZ, Ellis SR, Smith PW, Hallowell PT. Overwhelming Recurrent Clostridium difficile Infection after Reversal of Diverting Loop Ileostomy Created for Prior Fulminant C. difficile Colitis. Am Surg 2016;82:e194-5.

12. Hussain ZI, Todd N, Adams S, Stojkovic SG. Prevalence of clostridium difficile in excluded colons. Am Surg 2012;78:408-13.

13. Randall JK, Young BC, Patel G, Fitzgerald A, George BD. Is Clostridium difficile infection a particular problem after reversal of ileostomy? Colorectal Dis 2011;13:308-11.

14. Wilson MZ, Hollenbeak CS, Stewart DB. Impact of Clostridium difficile colitis following closure of a diverting loop ileostomy: results of a matched cohort study. Colorectal Dis 2013;15:974-81.

15. Zacharioudakis IM, Zervou FN, Pliakos EE, Ziakas PD, Mylonakis E. Colonization with toxinogenic C. difficile upon hospital admission, and risk of infection: a systematic review and meta-analysis. Am J Gastroenterol 2015;110:381-90;quiz 391.

16. Vonberg RP, Kuijper EJ, Wilcox MH, et al. Infection control measures to limit the spread of Clostridium difficile. Clin Microbiol Infect 2008;14 Suppl 5:2-20. 
17. Loo VG, Bourgault AM, Poirier L, et al. Host and pathogen factors for Clostridium difficile infection and colonization. $N$ Engl J Med 2011;365:1693-703.

18. Jump RL, Pultz MJ, Donskey CJ. Vegetative Clostridium difficile survives in room air on moist surfaces and in gastric contents with reduced acidity: a potential mechanism to explain the association between proton pump inhibitors and C. difficile-associated diarrhea? Antimicrob Agents Chemother 2007;51:2883-7.

19. Rubio-Perez I, Leon M, Pastor D, Diaz Dominguez J, Cantero R. Increased postoperative complications after protective ileostomy closure delay: An institutional study. World J Gastrointest Surg 2014;6:169-74.

20. Harries RL, Ansell J, Codd RJ, Williams GL. A systematic review of Clok ostridium difficile infection following reversal of ileostomy. Colorectal Dis 2017;19:881-7.

21. Hajibandeh S, Hajibandeh S, Sarma DR, et al. Meta-analysis of temporary loop ileostomy closure during or after adjuvant chemotherapy following rectal cancer resection: the dilemma remains. Int J Colorectal Dis 2019;34:1151-9.

22. Matsuda K, Hashiguchi Y, Tsukamoto M, et al. A case report of successful management of fulminant Clostridium difficile colitis postileostomy reversal with administration of vancomycin through a transverse colostomy. Surg Case Rep 2019;5:181. 